BULL. AUSTRAL. MATH. SOC.

VOL. $23(1981), 361-365$.

\title{
A CRITERION FOR THE HALL-CLOSURE OF FITTING. CLASSES
}

\author{
OWEN J, BRISON
}

\begin{abstract}
In a recent paper, Cusack has given a criterion, in terms of the Fitting class "join" operation, for a normal Fitting class to be closed under the taking of Hall $\pi$-subgroups. Here we show that Cusack's result can be slightly modified so as to give a criterion for any Fitting class of finite soluble groups to be closed under taking Hall $\pi$-subgroups.
\end{abstract}

\section{Introduction}

We will take our groups and classes of groups from the universe $\underline{\underline{S}}$ of all finite soluble groups. Let $\underline{\underline{F}}$ be a Fitting class and $\pi$ be a set of primes, and let $\underline{S}_{\pi}$ denote the class of all (finite, soluble) $\pi$-groups. Then $\underline{\underline{F}}$ is said to be Hall $\pi$-closed if whenever $G$ belongs to $\underset{F}{F}$, then the Hall $\pi$-subgroups of $G$ also belong to $\underline{\underline{F}}$. If we define $Y(\underline{S}, \underline{\underline{F}})$ to be the class of all those groups whose Hall $\pi$-subgroups belong to $\underline{\underline{F}}$, then it is clear that $\underline{\underline{F}}$ is Hall $\pi$-closed if and only if $\underline{F} \subseteq Y(\underline{S}, \underline{F})$. It is not hard to see that $Y\left(\underline{S}_{\pi}, \underline{F}\right)$ is itself a Fitting class. If $\underline{\underline{G}}$ is a further Fitting class, then the join, $\underline{\underline{F}} \vee \underline{\underline{G}}$, is the smallest Fitting class to contain both $\underline{\underline{F}}$ and $\underline{\underline{G}}$. In [6], Lockett associates with each Fitting class $\underline{F}$ the "new" Fitting classes $\underline{\underline{F}}^{*}$ and $\underline{\underline{F}} *$, and shows that $\underline{\underline{S}}_{*}$ is the so-called smallest normal Fitting class introduced in [2]. Then the result of Cusack in which we are interested is the following.

Received 28 November 1980. 
THEOREM [5, Theorem 5]. Let $\underline{\underline{F}}$ be a normal Fitting class and $\pi$ be a set of primes. Then $\underline{\underline{F}}$ is Hall $\pi$-closed if and only if

$$
\underline{\underline{F}}=\left(\underline{\underline{S}}_{\pi} \cap \underline{\underline{F}}\right) \vee\left(Y\left(\underline{\underline{S}}_{\pi}, \underline{\underline{S}}_{*}\right) \cap \underline{\underline{F}}\right) \text {. }
$$

\section{Preliminaries}

If $\underline{\underline{F}}$ and $\underline{\underline{G}}$ are Fitting classes, then $\underline{\underline{F G}}$ denotes the class

$$
\underline{\underline{\mathrm{FG}}}=\left(X \in \underline{\mathrm{S}}: X / X_{\underline{\underline{\mathrm{F}}}} \in \underline{\underline{\mathrm{G}}}\right),
$$

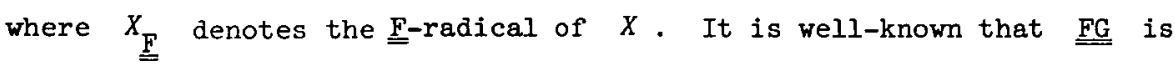
again a Fitting class.

We refer to [6] for the definitions of the classes $\underline{\underline{F}}^{*}$ and $\underline{\underline{F}}$ *; the following result, which is due to Lockett, collects the properties we need of these classes.

THEOREM 2.1 [6]. Let $\underline{\underline{F}}$ be a Fitting class and let $H \in \underline{\underline{F}}$. Then

(a) $\underline{\underline{F}}^{*}$ and $\underline{\underline{F}}_{*}$ are Fitting classes with $\underline{\underline{F}} \subseteq \underline{\underline{\mathrm{F}}} \subseteq \underline{\underline{F}}^{*}$;

(b) $H^{\prime} \leq H_{\underline{\underline{\mathrm{F}}}}$;

(c) $(H \times H)_{\underline{\underline{F}} *}=H_{\underline{\underline{F}}} \times H_{\underline{\underline{F}}}\left(\left(h^{-1}, h\right): h \in H\right) ;$ and

(d) if $\underline{\underline{\mathrm{G}}}$ is a further Fitting class then $(\underline{\underline{\mathrm{F}}} \cap \underline{\underline{\mathrm{G}}})^{*}=\underline{\underline{F}}^{*} \cap \underline{\underline{G}}^{*}$.

Recall that if $G$ and $H$ are groups and $N \leq G \times H$, then $N$ is said to be subdirect in $G \times H$ if $N(1 \times H)=G \times H=(G \times I) N$. It is clear that any subgroup of $H \times H$ which contains $\left.\left(h^{-1}, h\right): h \in H\right\rangle$ is subdirect in $H \times H$. We need the following result of Cusack.

THEOREM 2.2 [4, Corollary 2.6]. Let $\underline{\underline{U}}$ and $\underline{\underline{\mathrm{V}}}$ be Fitting classes such that $\underline{\underline{\mathrm{U}}} \subseteq \underline{\underline{\mathrm{V}}}^{*}$. Then a group $G$ lies in $\underline{\underline{\mathrm{U}}} \mathrm{v} \underline{\mathrm{V}}$ if and only if there exists a group $H \in \underline{\underline{\mathrm{U}}}$ such that $(G \times H)_{\underline{\underline{\mathrm{V}}}}$ is subdirect in $G \times H$.

The following facts about $Y(\underline{\underline{S}}, \underline{\underline{F}})$ can be found in [3] (where $Y(\underline{\underline{S}}, \underline{F})$ is called $\left.K_{\pi}(\underline{\underline{F}})\right)$. Note that part $(b)$ has also appeared in [1].

THEOREM 2.3. Let $\underline{\underline{F}}$ be a Fitting class, $\pi$ be a set of primes and $G$ be a group. Then 
(a) $G_{Y}\left(\underline{\underline{\mathrm{S}}}_{\pi}, \underline{\underline{\mathrm{F}})} \cap H=H_{\underline{\underline{\mathrm{F}}}}\right.$ for any Hazl $\pi$-subgroup $H$ of $G$;

(b) $Y\left(\underline{\underline{S}}_{\pi}, \underline{\underline{F}}^{*}\right)=\left(Y\left(\underline{\underline{S}}_{\pi}, \underline{\underline{F}}\right)\right)^{*}$;

(c) $Y\left(\underline{\mathrm{S}}_{\pi}, \underline{\mathrm{F}}\right)=Y\left(\underline{\mathrm{S}}_{-\pi}, \underline{\underline{\mathrm{F}}}\right) \underline{\mathrm{S}}_{\pi}$, ; and

(d) if $\underline{\underline{F}}$ is Hall $\pi$-closed, then so also are $\underline{\underline{F}}^{*}$ and $\underline{\underline{F}}$ *

\section{The theorem}

We model our proof on Cusack's; in particular, the three results below correspond, in order, to Lemma 3, Theorem 4 and Theorem 5 of [5]. The main difference is that here we use Theorem 2.3.

LEMMA 3.1. Let $\pi$ be a set of primes and $\underline{\underline{\mathrm{W}}}$ be a Hall $\pi$-closed Fitting class. Suppose that $G \in \underline{\underline{\mathrm{WS}}} \pi$ and that $H$ is a Hall $\pi$-subgroup

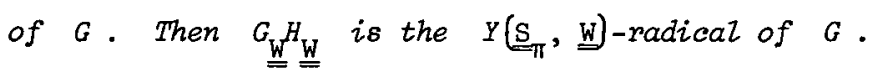

Proof. Let $\underline{\underline{Y}}$ denote $Y(\underline{\underline{S}}, \underline{W})$; then $G_{\underline{\underline{W}}} \leq G_{\underline{\underline{Y}}}$ since $\underline{\underline{W}}$ is hall $\pi$-closed. Now $G=G_{\underline{\underline{W}}} H$ by hypothesis, while $G_{\underline{\underline{\underline{Y}}}} \cap H=H_{\underline{\underline{W}}}$ by Theorem

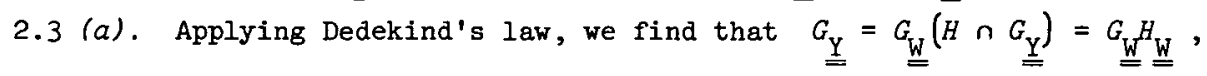
as claimed.

PROPOSITION 3.2. Let $\pi$ be a set of primes and $\underline{\mathrm{F}}$ be a Hall $\pi$-closed Fitting class. Then $\underline{\underline{F}}=\left(\underline{\underline{S}}_{\pi} \cap \underline{\underline{F}}\right) \vee\left(Y\left(\underline{\underline{S}}_{\pi}, \underline{\underline{F}}_{*}\right) \cap \underline{F}\right)$.

Proof. Let $\underline{\underline{Y}}$ denote $Y\left(\underline{S}_{\pi}, \underline{\underline{F}}_{*}\right)$. It follows from Theorem $2.3(\mathrm{c})$, (d) that $\underline{\underline{F}} \times \underline{\underline{S}}_{\pi}, \subseteq \underline{\underline{Y S}}, m=\underline{\underline{Y}}$, and so

$$
\underline{\underline{F}}+\underline{\underline{S}}_{\pi}, \cap \underline{\underline{F}} \subseteq \underline{\underline{Y}} \cap \underline{\underline{F}}
$$

Now let $G \in \underline{\underline{F}} \underline{\underline{S}} \underline{\underline{F}} \cap \underline{\underline{F}}$, and let $H$ be a Hall $\pi$-subgroup of $G$; then $H \in \underline{\underline{F}}$. Form $G \times H \in \underline{\underline{F}}$. Applying Lemma 3.1 with $\underline{\underline{W}}=\underline{\underline{F}} *$, and Theorem 2.1 (a) with $H \in \underline{\underline{F}}$, we find that

$$
\begin{aligned}
(G \times H)_{\underline{\underline{\mathrm{Y}}}} & =(G \times H)_{\underline{\underline{\mathrm{F}}} *}(H \times H)_{\underline{\underline{\mathrm{F}}} *} \\
& \geq\left(G_{\underline{\underline{\mathrm{F}}}} H_{\underline{\underline{\mathrm{F}}}} \times H_{\underline{\underline{\mathrm{F}}}}\right)\left(\left(h^{-1}, h\right): h \in H\right) .
\end{aligned}
$$

But clearly $G=G_{\underline{\underline{F}}} H$, and it follows that $(G \times H)_{\underline{\underline{Y}}}$ is subdirect in 
$G \times H$. Since $G \times H \in \underline{\underline{F}}$, this says that $(G \times H)_{\underline{\underline{Y}}}^{\underline{\underline{F}}}$ is subdirect in $G \times H$.

We now wish to apply Theorem 2.2 with $\underline{\underline{U}}=\underline{\underline{S}} \cap \underline{\underline{F}}$ and $\underline{\underline{V}}=\underline{\underline{Y}} \cap \underline{\underline{F}}$. Note that by Theorem 2.1 (d) and Theorem $2.3(b)$, (d), we have

$$
\underline{\underline{\mathrm{V}}}^{*}=\underline{\underline{\mathrm{Y}}}^{*} \cap \underline{\underline{\mathrm{F}}}^{*}=Y\left(\underline{\mathrm{S}}_{\pi}, \underline{\underline{F}}^{*}\right) \cap \underline{\underline{F}}^{*}=\underline{\underline{F}}^{*} \text {. }
$$

Thus $\underline{\underline{U}} \subseteq \underline{\underline{V}}^{*}$, and Theorem 2.2 implies that

$$
\underline{\underline{F}} \times \underline{\underline{S}} \rightarrow \underline{\underline{F}} \subseteq(\underline{\underline{S}} \cap \cap \underline{\underline{F}}) \vee(\underline{\underline{Y}} \cap \underline{\underline{F}}) .
$$

But it follows from Theorem 2.1 (b) that

$$
\underline{\underline{F}}=\left(\underline{\underline{F}} * \underline{\underline{S}}_{\pi} \cap \underline{\underline{F}}\right) \vee\left(\underline{\underline{F}} * \underline{\underline{S}}_{\pi}, \cap \underline{\underline{F}}\right),
$$

and so, combining (3.3) and (3.4), we conclude that

$$
\underline{\underline{F}}=(\underline{\underline{S}} \cap \underline{\underline{F}}) \vee(\underline{\underline{Y}} \cap \underline{\underline{F}}) \text {, }
$$

as required.

THEOREM 3.5. Let $\pi$ be a set of primes and $\mathrm{F}$ be a Fitting class. Then $\underline{\underline{F}}$ is Hall $\pi$-closed if and only if

$$
\underline{\underline{F}}=\left(\underline{S}_{\pi} \cap \underline{\underline{F}}\right) \vee\left(Y\left(\underline{S}_{\pi}, \underline{\underline{F}}_{*}\right) \cap \underline{\underline{F}}\right) \text {. }
$$

Proof. The "only if" assertion has been proved above. Thus suppose that $\left.\underline{\underline{F}}=\left(\underline{\underline{S}}_{\pi} \cap \underline{\underline{F}}\right) \vee\left(Y \underline{\underline{S}}_{\pi}, \underline{\underline{F}}_{*}\right) \cap \underline{\underline{F}}\right)$. Since $\underline{\underline{S}}_{\pi} \cap \underline{F} \subseteq Y\left(\underline{S}_{\pi}, \underline{E}\right)$, and since the operator $y\left(\underline{\underline{S}}_{\pi},\right)$ clearly respects inclusions, then Theorem 2.1 (a) implies that

$$
\underline{\underline{F}} \subseteq Y\left(\underline{S}_{\pi}, \underline{F}\right) \vee\left(Y\left(\underline{S}_{\pi}, \underline{\underline{F}}\right) \cap \underline{F}\right)=Y\left(\underline{S}_{\pi}, \underline{F}\right) .
$$

Thus $\underline{\underline{F}}$ is Hall $\pi$-closed, and the proof is complete.

\section{References}

[1] James C. Beidleman und Peter Hauck, "Über Fittingklassen und die Lockett-Vermutung", Math. 2. 167 (1979), 161-167.

[2] Dieter Blessenohl und Wolfgang Gaschütz, "Über normale Schunck- und Fittingklassen", Math. 2. 118 (1970), 1-8. 
[3] Owen J. Brison, "Hall operators for Fitting classes", Arch. Math. (Basel) 33 (1979), 1-9.

[4] Elspeth Cusack, "The join of two Fitting classes", Math. 2. 167 (1979), 37-47.

[5] Elspeth Cusack, "Normal Fitting classes and Hall subgroups", Bull. Austral. Math. Soc. 21 (1980), 229-236.

[6] F. Peter Lockett, "The Fitting class $\underline{F}^{*}$ ", Math. 2. 137 (1974), $131-136$.

Secção de Matemática Pura,

Faculdade de Ciências,

Avenida 24 de Julho, $134,3^{\circ}$,

1. 300 Lisboa,

Portugal. 